\title{
Cystic fibrosis transmembrane conductance regulator modulators: Present and future in cystic fibrosis treatment. A review
}

\author{
Diana De la Hoz, M.D. ${ }^{a}$, Milena Villamil Osorio, M.D. ${ }^{a, b}$ and \\ Sonia M. Restrepo-Gualteros, M.D. ${ }^{a, b}$
}

\begin{abstract}
Cystic fibrosis transmembrane conductance regulator (CFTR) modulators are the present and future of drug management for patients with cystic fibrosis. The objective of this article is to review this therapeutic option. Scientific articles were reviewed by searching the MedLine database, which is available through the Cystic Fibrosis Foundation's official website, from 2009 to 2018, in English. Twelve articles about the current status of research in CFTR modulators were selected without restrictions regarding the type of study. To date, the United States Food and Drug Administration has approved three modulators: ivacaftor, lumacaftor + ivacaftor, and tezacaftor + ivacaftor, while other 11 drugs are being studied in different investigation phases.CFTR modulator therapy is a developing reality aimed at the highest goal of personalized medicine and promises to improve the quality of life of cystic fibrosis patients.

Key words: cystic fibrosis, mutation, therapy, cystic fibrosis transmembrane conductance regulator.
\end{abstract}

http:/ / dx.doi.org/10.5546/aap.2019.eng.e131

To cite: De la Hoz D, Villamil Osorio M, RestrepoGualteros SM. Cystic fibrosis transmembrane conductance regulator modulators: Present and future in cystic fibrosis treatment. A review. Arch Argent Pediatr 2019;117(2):e131-e136.

a. Universidad

Nacional de

Colombia

Department of

Pediatrics, Bogotá

DC, Colombia.

b. Fundación Hospital

Pediátrico de la

Misericordia, Bogotá

DC, Colombia.

E-mail address:

Diana De la Hoz, M.D.: dcdeg@unal.edu.co

Funding:

None.

Conflict of interest:

None.

Received: 3-20-2018

Accepted: 10-20-2018
2012, the United States Food and Drug Administration (FDA) approved ivacaftor. Since then, more drugs aimed at correcting the protein defect at different points have been under study.

\section{Cystic fibrosis transmembrane conductance regulator PROTEIN}

The CFTR protein is a 1480 -amino acid glycoprotein comprised of 5 domains: 2 transmembrane domains, 2 nucleotide-binding domains, and 1 regulatory domain. ${ }^{2}$

To date, more than $1700 \mathrm{CF}$ causative mutations have been identified, grouped as follows:

- Class I mutation: This is a quantitative defect that prevents an adequate protein length, therefore resulting in a truncated protein; the mechanism corresponds to stop codon formation. The G542X mutation is the most common in this class. ${ }^{2-4}$

- Class II mutation: This is a quantitative defect with a protein folding problem that does not reach the surface because it suffers intracellular breakdown. The F508 mutation is the most common in this class. ${ }^{2-4}$

- Class III mutation: This is a qualitative defect. There is no response to cyclic adenosine monophosphate stimulation. The G551D mutation is the most common in this class. ${ }^{2-4}$

- Class IV mutation: This is a qualitative defect with a correctly localized protein but with an error in conductance. The R117H mutation is the most common one. ${ }^{2-4}$

- Class V mutation: It is seen as a reduced amount of CFTR at the membrane..$^{2-4}$ 
- Class VI mutation: There is an increase in the exchange of functional CFTR at the membrane..$^{2-4}$

\section{Cystic fibrosis transmembrane conductance regulator MODULATORS}

For the past 10 years, CF therapy has changed significantly with the emergence of CFTR modulators based on altered protein function as per the 6 mutation types described above. CFTR modulators are considered the ultimate goal of personalized medicine, according to which drug use depends on defect genotyping (Table 1). ${ }^{1-4}$

\section{Read-Through AGENTS}

Premature termination or nonsense mutations account for 5-10\% of all CFTR mutations and are class I. Ribosomal read-through agents are a type of drugs that suppress premature termination codons, thus allowing to obtain a normal length protein that may be transported to the surface.,3

Initially, the effect of low-dose aminoglycosides was studied in this group (the largest amount of evidence corresponds to gentamicin) and a significant reduction in the nasal potential difference was demonstrated. However, clinical benefits are limited due to the need of intravascular or intramuscular administration, the development of kidney toxicity, and the lack of power., ${ }^{2,3}$

Ataluren (PTC124) was a drug initially designed as a read-through agent but results were not as expected. In 2016, it was considered that the available evidence was insufficient to determine its effect on patients; on March $3^{\text {rd }}$, 2017, the company working on it announced it had stopped its development because no significant improvement in pulmonary function or in exacerbation reduction had been observed in the phase 3 clinical trial. The trial had started in 2015 with patients who had a class I mutation. ${ }^{5,6}$

To date, there are no drugs available for class I mutations, which are present in approximately $10 \%$ of CF patients. ${ }^{5,6}$

\section{Cystic fibrosis transmembrane conductance regulator POTENTIATORS}

Ivacaftor is the representative drug in this group. It was identified through a high performance selection process out of more than 228000 compounds, which was then used to achieve an adequate pharmacokinetic profile. ${ }^{3,7}$

In human bronchial epithelial cells with G551D/F508del mutations, ivacaftor has demonstrated to increase the liquid surface to levels similar to approximately half of the level observed in non-CF cells as well as the frequency of ciliary movement up to near normal values. ${ }^{7}$

Among ivacaftor studies, it is worth noting two phase 3 randomized, double-blind, controlled studies: ENVISION and STRIVE. Both studies showed a significant reduction in sweat chloride. In addition, they showed an absolute increase in forced expiratory volume in 1 second $\left(\mathrm{FEV}_{1}\right)$ of $10.4 \%$ (STRIVE) and $10.7 \%$ (ENVISION). Ivacaftor also caused a significant reduction of $55 \%$ in pulmonary exacerbations in the STRIVE study. A subsequent study, called PERSIST, included 144 adolescents / adults and 48 children with the G551D mutation who had been previously treated with ivacaftor as part of the STRIVE and ENVISION studies, and demonstrated a sustained improvement of pulmonary function, weight, and pulmonary exacerbations up to 144 weeks of treatment. ${ }^{3,4}$

It is worth noting that the beneficial effect of ivacaftor is not limited to the G551D mutation; it extends to a wide number of mutations, including

TABLE 1. Class mutations and therapy examples

\begin{tabular}{|c|c|c|c|c|c|c|}
\hline & Class I & Class II & Class III & Class IV & Class V & Class VI \\
\hline Mutation type & $\begin{array}{l}\text { No protein } \\
\text { synthesis } \\
\text { Nonsense, } \\
\text { frameshift. }\end{array}$ & $\begin{array}{l}\text { Folding } \\
\text { defect } \\
\text { Missense, } \\
\text { deletion. }\end{array}$ & $\begin{array}{l}\text { Channel } \\
\text { opening } \\
\text { defect } \\
\text { Missense, } \\
\text { Amino acid } \\
\text { change. }\end{array}$ & $\begin{array}{l}\text { Ion } \\
\text { transport } \\
\text { defect } \\
\text { Missense, } \\
\text { Amino acid } \\
\text { change. }\end{array}$ & $\begin{array}{l}\text { Protein } \\
\text { synthesis } \\
\text { reduction } \\
\text { Splicing. }\end{array}$ & $\begin{array}{l}\text { Protein } \\
\text { half-life } \\
\text { reduction } \\
\text { Missense, } \\
\text { Amino acid } \\
\text { change. }\end{array}$ \\
\hline Therapy & $\begin{array}{l}\text { Gene } \\
\text { therapy }\end{array}$ & $\begin{array}{l}\text { Lumacaftor, } \\
\text { Ivacaftor, } \\
\text { Tezacaftor } \\
\text { Gene therapy }\end{array}$ & $\begin{array}{l}\text { Ivacaftor } \\
\text { Gene } \\
\text { therapy }\end{array}$ & $\begin{array}{l}\text { Ivacaftor } \\
\text { Gene therapy } \\
\text { Tezacaftor } \\
\text { (specific } \\
\text { mutations) }\end{array}$ & $\begin{array}{l}\text { Gene therapy } \\
\text { Tezacaftor } \\
\text { (specific } \\
\text { mutations) }\end{array}$ & $\begin{array}{l}\text { Gene } \\
\text { therapy }\end{array}$ \\
\hline
\end{tabular}


G551S, G1349D, G1244E, G970R, G178R, S549N, S549R, S1251N, and S1255P, among others. In turn, such potentiating effect even comprises other class mutations, such as class IV mutations $\mathrm{R} 117 \mathrm{H}$ and D1152H, which have shown a favorable response. ${ }^{4}$

In May 2017, the FDA approved this drug for 23 other mutations for which studies demonstrated a benefit. At present, its use is being studied in patients with 6 other mutations included in class $\mathrm{V}$ (no specific drug has been developed yet for all mutations in this group). ${ }^{8}$

\section{Cystic fibrosis transmembrane conductance regulator CORRECTORS - COMBINED THERAPY}

These drugs are designed to increase the amount of "functional" protein that reaches the apical cell membrane. The target of this group of drugs are class II mutations, such as F508del. ${ }^{2,3}$

Lumacaftor (VX-809) is a molecule that evidenced, in in vitro studies, a 7.1 -fold improvement in CFTR maturation together with a 5-fold improvement in chloride transport. It appears that lumacaftor acts at the level of the first transmembrane domain (membrane-spanning domain, MSD), which leads to a partial correction of the CFTR folding defect by improving interaction between the MSD1 and MSD2 transmembrane domains and the NBD1 nucleotide-binding domain. ${ }^{4}$ Lumacaftor monotherapy did not show significant differences in the sweat test, $\mathrm{FEV}_{1}$ values, the Revised Cystic Fibrosis Quality of Life Questionnaire in Adolescents and Adults (CFQ-R) or the nasal potential difference. ${ }^{9}$

One of the first studies that demonstrated the effectiveness of a combined therapy included three successive cohorts; cohort 1 included 64 patients homozygous for F508del mutation, whereas cohorts 2 and 3 included a total of 124 patients both homozygous and heterozygous for the same mutation. Treatment with lumacaftor + ivacaftor $(\mathrm{L}+\mathrm{I})$ reduced the mean level of sweat chloride in $9.1 \mathrm{mmol} / \mathrm{L}(p<0.001)$ in cohort 1 but did not cause a significant reduction in cohorts 2 and 3 . In turn, homozygous patients (especially those in cohort 2) showed an improvement in $\operatorname{FEV}_{1}(p=0.013)$, but this change was not observed in patients who were heterozygous for the same mutation. Finally, it was concluded that the combined therapy improved $\mathrm{FEV}_{1}$ in CF patients homozygous for the F508del mutation, with a moderate effect on the level of sweat chloride. ${ }^{10}$

The results of the TRAFFIC and TRANSPORT studies were published in 2015 and documented the safety and effectiveness of the combined therapy. These studies included 1108 patients older than 12 years who were homozygous for the F508del mutation. The primary endpoint was an absolute change in $\mathrm{FEV}_{1}$ at week 24. Both studies showed significant improvements in the primary endpoint; the difference between the combined therapy and placebo in terms of change in the expected $\mathrm{FEV}_{1}$ ranged from 2.6 to 4.0 percentage points $(p<0.001)$. The rate of pulmonary exacerbations was 30-39\% lower in the group treated with $\mathrm{L}+\mathrm{I}$ than in the placebo group. ${ }^{11}$

An observational, multicenter study assessed the effects of the combined therapy with $\mathrm{L}+\mathrm{I}$ by measuring the number of adverse events, treatment discontinuation, $\mathrm{FEV}_{1}$, and body mass index (BMI) at months 1 and 3 after starting lumacaftor + ivacaftor in adults with $\mathrm{CF}$ and a $\mathrm{FEV}_{1}$ below $40 \%$ of the predicted value. The study demonstrated that $51 \%$ of patients receiving $\mathrm{L}+\mathrm{I}$ had adverse events leading to treatment discontinuation in at least $24 \%$ of them. However, those who continued with lumacaftor + ivacaftor showed a significant improvement in $\mathrm{FEV}_{1}(+3.19 \%$ at 3 months), which was similar to what was observed in patients recruited in phase 3 clinical trials and which may have very good results, even in patients with a $\mathrm{FEV}_{1} \leq 30 .{ }^{12}$

Tezacaftor is another modulator that facilitates CFTR processing and intracellular transport to increase the amount of protein delivered to the cell surface, therefore promoting ivacaftor action; besides, it appears to have a better safety profile compared to lumacaftor. In November 2017, two phase 3 studies were published that demonstrated the safety and effectiveness of combined tezacaftor + ivacaftor $(\mathrm{T}+\mathrm{I}) \cdot{ }^{13,14}$

The EVOLVE study was conducted in 510 patients homozygous for the F508del mutation. The absolute and relative change in the $\mathrm{FEV}_{1}$ percentage in favor of $\mathrm{T}+\mathrm{I}$ compared to placebo was 4 and 6.8 percentage points, respectively, and the rate of pulmonary exacerbations was $35 \%$ lower in the $\mathrm{T}+\mathrm{I}$ group compared to the placebo group $(p=0.005){ }^{13}$

The EXPAND study included 248 patients older than 12 years who were heterozygous for the F508del mutation and had a CFTR mutation associated with the channel's residual function. Interventions were analyzed in patient groups, 162 received tezacaftor-ivacaftor; 157, ivacaftor alone; and 162, placebo. The change in $\mathrm{FEV}_{1}$ (from baseline to the average of week 4 and 
week 8) was 6.8 percentage points for $\mathrm{T}+\mathrm{I}$ and 4.7 percentage points for ivacaftor alone $(p<0.001$ for both comparisons). The CFQ-R score also favored active treatment groups significantly. The incidence of adverse events was similar in all intervention groups. ${ }^{14}$ The tezacaftor + ivacaftor combination was approved by the FDA in February 2018 for its use in patients homozygous for the F508del mutation or with a single copy of 26 specific mutations with residual protein activity. ${ }^{15}$

\section{THE CHALLENGE OF CREATING Cystic fibrosis transmembrane conductance regulator MODULATORS}

To date, there are 3 CFTR modulators approved for their use in children (ivacaftor, ivacaftor + lumacaftor, and tezacaftor + ivacaftor) and 11 are still under development. So, what is the reason why there are not more CFTR modulators available? The answer lies in the difficulty entailed by conducting, for certain, each study phase, from preclinical to late phase clinical trials.

\section{Preclinical phase, the early task}

Implementing a therapy with CFTR modulators requires the genetic analysis of the protein defect, and many times this is not available for all patients or even in all health systems. ${ }^{16}$

- If a new modulator is being developed for a mutation that has already been tested with a different drug, the problem lies in making long-term placebo-controlled comparisons. ${ }^{16}$

- Modulators should be assessed in preclinical trials to establish if their observed activity is associated with a level of CFTR modulation that is probably equal to or higher than the therapies currently approved so that they receive financial support or raise concern in the patient community which, in the case of $\mathrm{CF}$, has mobilized a great deal of interest in new therapy research. ${ }^{16}$

\section{Early phase studies, biomarkers, and safety}

- The safety assessment should prove that all components and quantities used are indispensable to achieve the corresponding effectiveness for the dosage of the studied modulator. ${ }^{16}$

- Biomarkers should be selected so that the lowest number of subjects possible is included in the sample and so that they allow to predict a clinically significant effect in subsequent studies with a larger sample. In this regard, 3 biomarkers are currently used: nasal potential difference, sweat chloride, and duodenal $\mathrm{pH}$; of these, sweat chloride appears to be the most sensitive one with the greatest performance scope. ${ }^{16}$

- Measuring sweat chloride has relevant characteristics: it is a standardized, noninvasive test with low variability (which is key for extrapolation to larger populations) and high sensitivity. Although it does not predict individual $\mathrm{FEV}_{1}$ values, there is strong evidence that relates sweat chloride to considerable changes in $\mathrm{FEV}_{1}$ at the population level. ${ }^{16}$

- A tudy published in 2017 proposed that the functional restoration of the CFTR channel, measured in human nasal epithelium cultures, may predict long-term clinical benefits. The study used nasal potential difference and CFTR expression (measured by fluorescence) in human nasal epithelium cultures as biomarkers instead of bronchial epithelium cultures. The results demonstrated that the level of CFTR correction correlated significantly to the change in $\mathrm{FEV}_{1}$ at 6 months in 8 patients treated with CFTR modulators. ${ }^{17}$

\section{Late phase studies, demonstration of clinical effectiveness}

- Researchers must develop studies in compliance with ethics, especially when there is no therapy approved as a standard of care. The discussion revolves around the conduct of placebo-controlled studies. This is because active-comparator studies should be proposed only after determining that there is not an appropriate treatment-naïve population for which a placebo-controlled trial could be conducted (e.g., international populations for which the drug is not approved or available). In addition, they may be developed when patients or treating physicians are not against withdrawal from current standard of care and assignment to placebo for a given duration. ${ }^{16}$

- If the decision gravitates towards activecomparator trials, three potential challenges should be faced: I) an increased sample size is required compared to placebocontrolled studies; II) potential costs incurred for acquisition and blinding of the active comparator; III) a less clear comparator group for interpreting safety data. ${ }^{16}$ 
- The main objective of these studies will be a significant change in pulmonary function tests, which may be considered a major challenge. ${ }^{16}$

\section{WHAT IS THE FUTURE OF Cystic fibrosis transmembrane conductance regulator MODULATORS?}

The current status of CFTR modulator research is extensively described in the Cystic Fibrosis Foundation's website, where they describe ivacaftor, lumacaftor + ivacaftor, and tezacaftor + ivacaftor and other 11 modulators under development in different study phases.

- Two are in phase 3:

Tezacaftor (VX-661) + ivacaftor + VX-445

Tezacaftor (VX-661) + ivacaftor + VX-659

- Five are in phase 2: QBW251, FDL169, GLPG2222, PTI-428 (amplifier), vx-561.

- And 4 are in phase 1: PTI-801, PTI-808, QR-010, MRT2005.

\section{CONCLUSION}

The worldwide scientific community is greatly committed to the study of new treatments for CF patients. CFTR modulator therapy is a developing reality targeted at personalized medicine, which very specifically addresses the genetics of each CF patient who is a candidate for treatment. The drugs developed to date represent a challenge for clinical practice but there is hope for better results in the future.

\section{Acknowledgments}

Department of Pediatrics of Universidad Nacional de Colombia, Division of Pediatric Pulmonology of Hospital de la Misericordia.

\section{REFERENCES}

1. Zemanick E, Ong T, Daines C, Dellon E, et al. Highlights from the 2015 North American Cystic Fibrosis Conference. Pediatr Pulmonol. 2016; 51(6):650-7.

2. Derichs N. Targeting a genetic defect: cystic fibrosis transmembrane conductance regulator modulators in cystic fibrosis. Eur Respir Rev. 2013; 22(127):58-65.

3. Solomon S, Marshall S, Ramsey B, Rowe S. Breakthrough Therapies: Cystic Fibrosis (CF) Potentiators and Correctors. Pediatr Pulmonol. 2015; 50(Suppl 40):S3-13.

4. Barry P, Ronan N, Plant B. Cystic Fibrosis Transmembrane Conductance Regulator Modulators: The End of the
Beginning. Semin Respir Crit Care Med. 2015; 36(2):287-98.

5. Aslam AA, Higgins C, Sinha IP, Southern KW. Ataluren and similar compounds (specific therapies for premature termination codon class I mutations) for cystic fibrosis. Cochrane Database Syst Rev. 2017; 1:CD012040.

6. Cystic Fibrosis Foundation. Drug Company Ends Ataluren Program forCFNonsenseMutations (Bethesda:March3,2017) [Internet]. [Accessed on: June $\left.4^{\text {th }}, 2017\right]$. Available at: www. cff.org/ News/News-Archive/2017/Drug-Company-EndsAtaluren-Program-for-CF-Nonsense-Mutations/.

7. Van Goor F, Hadida S, Grootenhuis PD, Burton B, et al. Rescue of CF airway epithelial cell function in vitro by a CFTR potentiator, VX-770. Proc Natl Acad Sci U S A. 2009; 106(44):18825-30.

8. Cystic Fibrosis Foundation. FDA Approves Ivacaftor for 23 Additional CFTR Mutations (Bethesda: May 17, 2017) [Internet]. [Accessed on: June $5^{\text {th }}$, 2017]. Available at: www. cff.org/News/ News-Archive/2017/FDA-Approves-Ivacaftorfor-23-Additional-CFTR-Mutations / .

9. Clancy JP, Rowe SM, Accurso FJ, Aitken ML, et al. Results of a phase IIa study of VX-809, an investigational CFTR corrector compound, in subjects with cystic fibrosis homozygous for the F508del-CFTR mutation. Thorax. 2012; 67(1):12-8.

10. Boyle MP, Bell SC, Konstan MW, McColley SA, et al. A CFTR corrector (lumacaftor) and a CFTR potentiator (ivacaftor) for treatment of patients with cysticfibrosis whohavea phe508del CFTR mutation: a phase 2 randomised controlled trial. Lancet Respir Med. 2014; 2(7):527-38.

11. Wainwright CE, Elborn JS, Ramsey BW, Marigowda G, et al. Lumacaftor-ivacaftor in patients with cystic fibrosis homozygous for Phe508del CFTR. N Engl J Med. 2015; 373(3):220-31.

12. Hubert D, Chiron R, Camara B, Grenet D, et al. Real-life initiation of lumacaftor/ivacaftor combination in adults with cystic fibrosis homozygous for the Phe508del CFTR mutation and severe lung disease. J Cyst Fibros. 2017; 16(3):388-91.

13. Taylor-Cousar JL, Munck A, McKone EF, van der Ent CK, et al. Tezacaftor-Ivacaftor in Patients with Cystic Fibrosis Homozygous for Phe508del. NEnglJMed.2017;377(21):201323.

14. Rowe SM, Daines C, Ringshausen FC, Kerem E, et al. Tezacaftor-Ivacaftor in Residual-Function Heterozygotes with Cystic Fibrosis. N Engl J Med. 2017; 377(21):2024-35.

15. Cystic Fibrosis Foundation. FDA Approves New CFTR Modulator Treatment for Cystic Fibrosis (Bethesda: Feb 12, 2018) [Internet]. [Accessed on: July $12^{\text {th }}, 2018$ ]. Available at: https:/ / www.cff.org/News/ News-Archive/2018/FDAApproves-New-CFTR-Modulator-Treatment-for-CysticFibrosis/.

16. Mayer-Hamblett N, Boyle M, VanDevanter D. Advancing clinical development pathways for new CFTR modulators in cystic fibrosis. Thorax. 2016; 71(5):454-61.

17. Pranke IM, Hatton A, Simonin J, Jais JP, et al. Correction of CFTR function in nasal epithelial cells from cystic fibrosis patients predicts improvement of respiratory function by CFTR modulators. Sci Rep. 2017; 7(1):7375. 